\title{
Tumor neuroectodérmico pigmentado infantil. Reporte de un caso
}

\section{Neuroectodermal tumor pigmented children. A case report}

\author{
Díaz Caballero AJ*, Cantillo Pallares O**, Herrera Herrera A***
}

\section{RESUMEN}

El tumor neuroectodérmico pigmentado es un tumor benigno de crecimiento rápido e intensamente pigmentado del maxilar inferior (y en ocasiones de otros sitios), que consiste en una masa infiltrativa de células dispuestas según un patrón alveolar. Aparece casi exclusivamente en lactantes. Se presenta como una tumoración protuberante en un maxilar o la mandíbula principalmente en tejidos blandos; encontrándose excepcionalmente en el tracto intestinal, pelvis, retroperitoneo y riñón. Clínicamente se manifiesta como una tumoración localizada, con presencia o no de zonas pigmentadas.

Estas neoplasias son raras y afectan mayormente niños, que por lo general son menores de un año y que pueden ser congénitos. Su localización en cerca de un $70 \%$ es la mandíbula; facio-cervical, en $90 \%$, también se reportaron en localizaciones como: fontanela anterior, hueso temporal y duramadre, epidídimo, huesos largos, mediastino. Éstos son considerados como formas periféricas, ya que existen formas centrales que pueden afectar cerebro, cerebelo, glándula pineal.

La clínica de estos casos puede simular a muchas otras patologías, dificultando su diagnostico ya que es una masa que protruye y deforma que rara vez ulcera. Las radiografías muestran una masa radiolúcida con capacidad de destrucción focal y desplazamiento de los dientes, por lo que se puede confundir con un ameloblastoma es por esto que es necesario un manejo adecuado con los exámenes histológicos necesarios como se hizo en el presente caso.

Palabras clave: Tumores neuroectodérmicos, neoplasias.

\section{SUMMARY}

The Pigmented neuroectodermal tumor is a benign tumor of intensely pigmented and rapid growth of the lower jaw (and in occasions of other sites [places]), that consists of a mass of infiltrative cells arranged according to an alveolar boss. It (he, she) appears almost exclusively in nursing. He (she) appears as a protruding tumor in the jaw Maxillary one or principally in the soft (smooth) fabrics; being exceptionally in the intestinal tract, pelvis, retroperitoneum and kidney. Clinical, it (he, she) demonstrates located as a tumor or not with presence of pigmented areas.

These tumors are rare and mostly affect children, who are generally under one year and can be congenital. Its location in about 70 percent is the mandible, facio-cervical in 90 percent have also been reported in locations such as anterior fontanel, temporal bone and dura, epididymis, long bones, mediastinum. These are regarded as peripherally, as there are ways that can affect Central Brain, cerebellum, pineal gland.

The clinic in these cases may mimic other pathologies, easily diagnosed as a protruding mass that deforms and rarely ulcerates. Radiographs showed a radiolucent mass capable of focal destruction and displacement of

Odontólogo, Universidad de Cartagena. Especialista en Periodoncia. Universidad Javeriana. Profesor titular, Universidad de Cartagena. Director Grupo de Investigación GITOUC.

** Odontólogo, Universidad de Cartagena, Cirujano Máxilo Facial. Universidad de Buenos Aires. Argentina. Profesor titular, Universidad de Cartagena.

*** Estudiante VIII semestre, Facultad de Odontología. Universidad de Cartagena. Estudiante investigadora Grupo de investigación GITOUC. 
teeth, it can be confused with an ameloblastoma that is why it is necessary to an adequate histological examinations were made as necessary in this case.

Key words: Neuroectodermal tumors, neoplasms.

Fecha de recepción: 20 de diciembre de 2009.

Aceptado para publicación: 20 de enero de 2010.

Díaz Caballero AJ, Cantillo Pallares O, Herrera Herrera, A. Tumor neuroectodérmico pigmentado infantil. Reporte de un caso. Av. Odontoestomatol 2011; 27 (4): 189-194.

\section{INTRODUCCIÓN}

El tumor neuroectodérmico melanótico de la infancia es una neoplasia poco frecuente, descrita por primera vez, en la literatura alemana, por Krompecher (3) en 1918, con el nombre de melanocarcinoma congénito. Posteriormente, una gran cantidad de nombres se utilizaron para denominar esta neoplasia: progonoma melanótico de la infancia, odontoma melanótico epitelial, adamantinoma pigmentado, ameloblastoma melanótico, épulis pigmentado congénito, melanoameloblastoma, melanocitoma, teratoma retinal o teratoma retinoblastico y, finalmente, fueron descritos y clasificados hasta 1963 el término más aceptado en la actualidad, tumor neuroectodérmico melanótico de la infancia (4).

Como su nombre lo indica, el tumor se presenta predominantemente durante la etapa de lactante menor; y entre los hallazgos clínicos se presenta como una tumoración protuberante en un maxilar o la mandíbula. La piel o la mucosa se presentan muy distendidas sobre la lesión, pero nunca o muy raramente se ulcera. Desde el punto de vista radiológi$\mathrm{co}$, el tumor es una lesión quística radio lúcida con capacidad de destrucción local y que puede desplazar los dientes en desarrollo; el 92\% de los casos reportados se diagnosticaron en menores de 12 meses de edad, con igual frecuencia en ambos géneros. Clínicamente se manifiesta como una tumoración localizada, con presencia o no de zonas pigmentadas (5).

La agresividad y el desenlace de ésta patología requiere un diagnóstico temprano y acertado, diferenciándolo de algunas entidades clínicas similares como: neuroblastoma (segunda neoplasia sólida extracraneal más común), sarcoma de Ewing's, tumor de Wilms, neuroepitelioma y otros tumores de células redondas $(6,7)$.

El examen histológico macroscópicamente, el tumor tiene un color que oscila entre gris pizarra y el azul negruzco dependiendo de la cantidad de pigmento melánico. Esta formado por espacios alveolares irregulares revestidos de células cúbicas que contienen cantidades variables de pigmentos melánicos $(8,9)$.

La histogenia de este tumor es un motivo de controversia. Las ideas de que se tratara como un melanoma congénito o de un tumor odontógeno se abandonaron por varias razones. El primero no justifica el componente neuroblástico y el segundo no explica los tumores que se desarrollan en lugares donde no hay restos odontógenos. En la actualidad, la teoría mas atrayente es que el tumor deriva de la cresta neural, ya que permite aclarar la distribución de las lesiones, justifica la presencia de elementos pigmentarios y neuroblásticos y explica los raros casos asociados al aumento de las concentraciones de ácido vanilmandélico (10).

\section{CASO CLÍNICO}

Paciente de 6 meses de edad de sexo masculino, de procedencia rural, que acude con sus padres al hospital de atención pediátrica, por abultamiento y deformidad facial, a nivel del tercio medio de la cara en la hemifacial derecha desde que nació de unos 4 meses de evolución. Entre sus antecedentes médi- 
co-personales no presentaba datos de relevancia alguna.

Al examen clínico extraoral, encontramos al paciente con asimetría facial y aumento marcado del tercio medio derecho de la cara, al examen intraoral se observa un tumor que abarca la región anterior y media del maxilar superior derecho y parte del izquierdo, de mas o menos 7 u $8 \mathrm{~cm}$ de diámetro mayor, por 3 o $4 \mathrm{~cm}$ de alto y que se extiende hasta la parte posterior del paladar duro con la respectiva deformación anatómica del maxilar superior, influyendo también sobre el maxilar inferior. De consistencia blanda y recubierto por mucosa de color violáceo, lisa, brillante, asintomático, no relacionada con trauma o proceso infeccioso (Figura 1).

Al examen radiográfico, con tomografía axial computarizada, observamos una lesión intraósea, que expande y desplaza corticales, que ocupa la región anterior y media del maxilar superior derecho, de aproximadamente 6 ó $7 \mathrm{~cm}$ de diámetro mayor por 3,5 ó 4 $\mathrm{cm}$ de alto, con bordes bien definidos (Figura 2).

Con la impresión clínica y la radiografía se podía sospechar que consistiera en un tumor neuroectodérmico pigmentado de la infancia o de un adenoma pleomórfico, por la consistencia blanda, la forma y ubicación. Para esto se realizo una biopsia

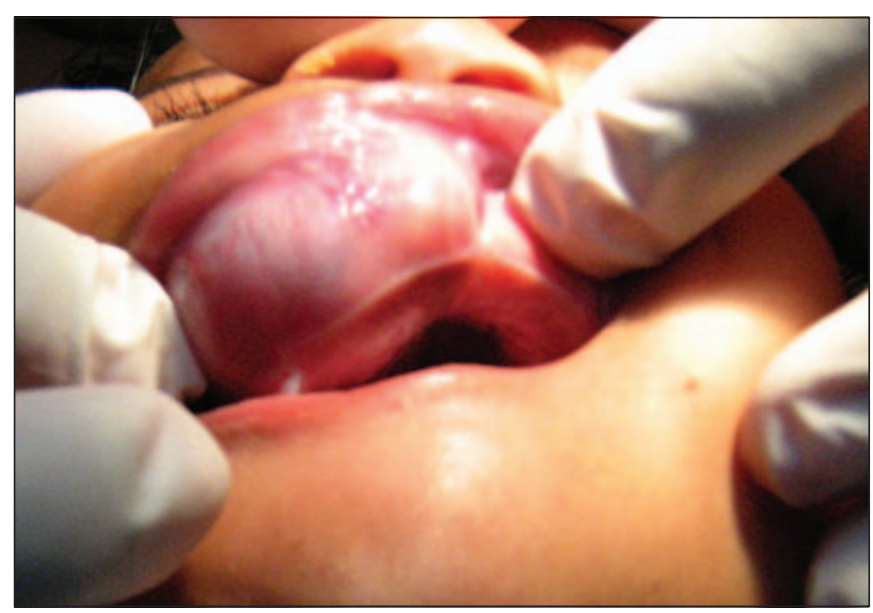

Fig. 1. Se observa un tumor que abarca la región anterior y media del maxilar superior derecho y parte del izquierdo, que se extiende hasta la parte posterior del paladar duro con la respectiva deformación anatómica del maxilar superior, influyendo también sobre el maxilar inferior.

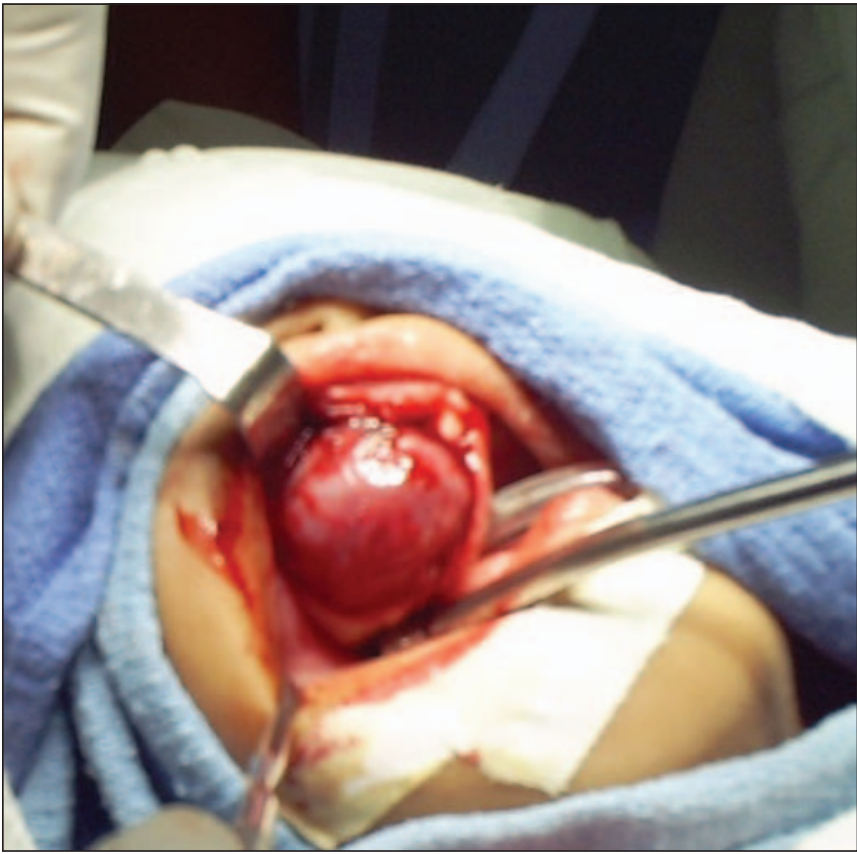

Fig. 2. Se observa una lesión intraósea, que expande y desplaza corticales, que ocupa la región anterior y media del maxilar superior derecho, con bordes bien definidos.

aspirativa con aguja fina (BACAF) Para descartar o confirmar contenido líquido de la lesión obteniendo los resultados negativo para dicha prueba y solo se obtuvo líquido hemático confirmando un tumor neuroectodérmico pigmentado de la infancia.

Para el plan de tratamiento según la impresión clínica, se procede a hacer la eliminación o la escisión total del tumor bajo anestesia general y luego enviar a patología el espécimen para obtener un diagnostico de certeza.

El procedimiento se realizó bajo anestesia general con intubación orotraqueal. Previa asepsia y antisepsia se realizó una incisión sobre la lesión a nivel del reborde alveolar y que luego se procedió a disecar la lesión siguiendo zona de clivaje (Figura 3). Se realiza la escisión completa de la lesión encapsulada con hemostasia, posteriormente se coloca gasa yodoformada y se sutura con vicryl $4-0$, sin complicaciones.

Se dejó hospitalizado por 3 días para manejo postoperatorio inmediato y posteriores controles por consulta externa. 


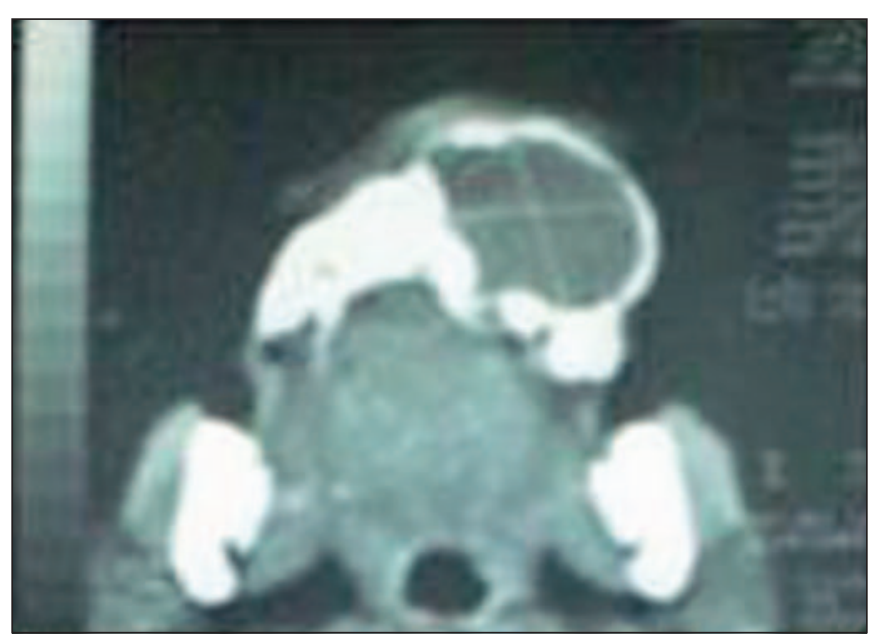

Fig. 3. Se observa la escisión completa de la lesión encapsulada.

El espécimen quirúrgico llevado a patología, confirmó el diagnostico inicial (Figuras 4 y 5).

\section{DISCUSIÓN}

Enzinger y Weiss en 1995 mencionan que el tumor neuroectodérmico pigmentado recibe históricamente diferentes denominaciones tales como progronoma melanótico o tumor del esbozo de la retina, entre otros y que se acepta generalmente como una neoplasia benigna de localización en los maxi- lares superiores como se observa en el examen histológico del paciente reportado en el presente artículo (11).

En cuanto al diagnóstico, es importante diferenciar el tumor neuroectodérmico pigmentado de otras patologías tales como: tumor de Wilms, angiomiolipoma, linfoma maligno, entre otros, por cuanto el abordaje terapéutico y los resultados del mismo son bien diferentes en estas entidades, es por esto que se debe realizar un buen examen clínico previo como se realizo en el caso observando la anatomía, la consistencia, realizando BACAF para observar el contenido dentro de la lesión y confirmar con el estudio de patología. La inmunohistoquímica es una buena forma para confirmar que se trata inequívocamente de un tumor originado y relacionado con la cresta neural (neuroectodérmico) (12).

Basados en el caso que se reporta y por los otros casos de la literatura, considera que el tumor neuroectodérmico pigmentado constituye una entidad clínica única, muy poco reportado en la literatura, que constituye uno de los problemas mas grandes en los niños, que histológicamente demostró estar formado por células pequeñas redondas dispuestas en grupos e hileras, separadas por abundante tejido fibroso, además se observaron células epiteliales de disposición lobular, predominantemente con pigmen-
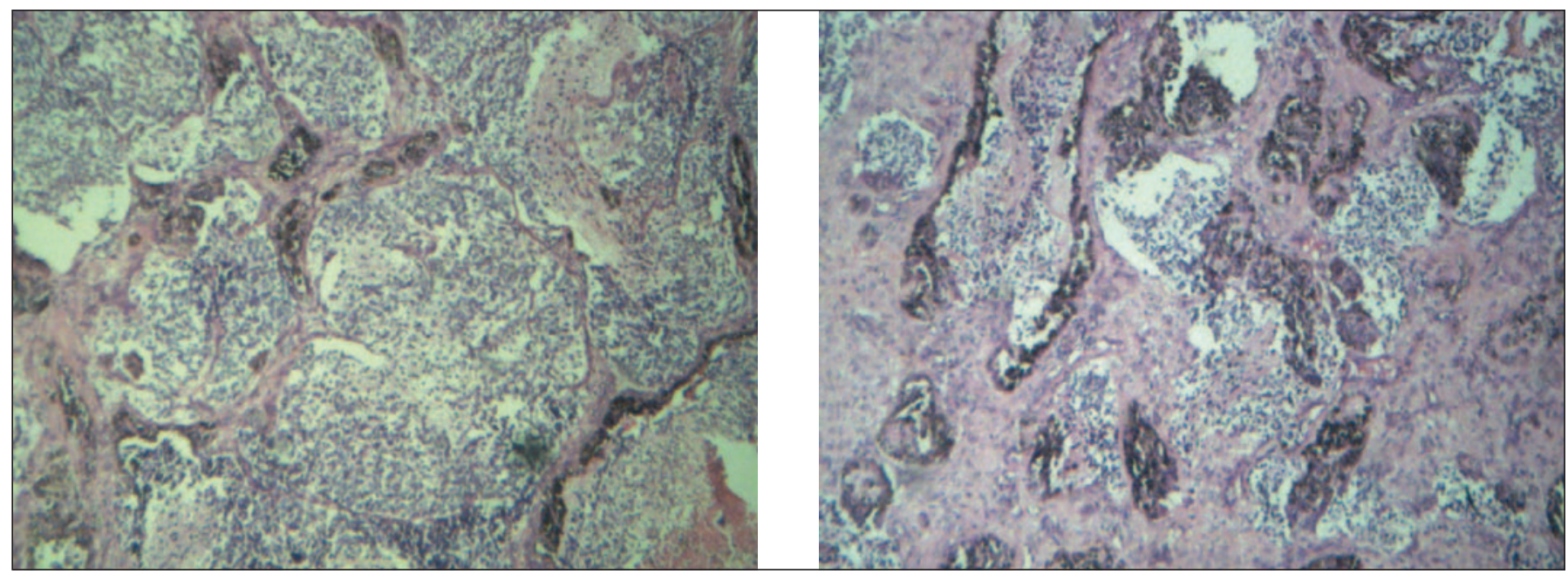

Figs. 4 y 5. Se observan espacios alveolares irregulares revestidos de células cúbicas que contienen cantidades variables de pigmentos melánicos, de color que oscila entre gris pizarra y el azul negruzco. 
tos melánicos en su citoplasma que la hace diferenciar de las otras patologías, lo que permite establecer una gran concordancia con el resultado histológico del caso que se reporta (13).

Aunque benignos, son localmente agresivos; algunos autores como Dilu et al, y Bardfield y Pleassants refieren que tras la extirpación no hay evidencias de recidivas $(14,15)$, sin embargo en la serie revisada por el Instituto de Patología de las Fuerzas Armadas de Washington se difiere y se reportan que cerca de la mitad recurren y aproximadamente del cinco al diez por ciento pueden producir metástasis $(16,17)$, por lo que se impone una amplia extirpación y un seguimiento estrecho, tal como se hizo en el caso actual.

\section{BIBLIOGRAFÍA}

1. Marracki R, Ben Romdhane K, Kharrat N, Sioud H. Melanotic progonoma in an infant. A propos of a case. Rev Stomatol Chir Maxilofac 1988; 89 (5):316-9.

2. Rickert CH, Probst-Cousin S, Blasius S, Gullotta E. Melanotic progonoma of the brain: A ccase report and review. Child's nervous system 1998; 14(8):389-93.

3. Krompecher, E. Sur Histogenese und Morphologie der Adamantinome und sonstiger Kiefergeschwulste. Beitr Path Anat 1918;64: 165.

4. Luire HI. Congenital Melanocarcinoma, Melanotic Adamantinoma, Retinal Anlage Tumor, Progonoma, and Pigmented Epulis of Infancy, Cancer 1961;14:1090.

5. Rubistein L. Tumors and tumors-like lesions of maldevelopmental origin, in Tumors of The Central Nervous System, ed, Rubistein L, 2nd edit, Armed Forces Institute of Pathology, Washington 1985;720-2.

6. Rickert CH, Probst-Cousin S, Blasius S, Gullotta E. Melanotic progonoma of the brain: A ccase report and review. Child's nervous system. 1998; 14(8):389-93.

7. Claros P, Claros A, Claros A Jr, Clavería A. Progonoma Melanótico del maxilar: Presentación de un caso. Acta Otorrinolaringol Esp. 1990;41 (2):103-6.

8. Porter JE, Cummings, GO Jr. Melanotic Progonoma of the Maxila Archives of Pathology 1963;76:3.

9. Stiller U, Herrmann P, Holzhausen HJ. Melanocitic neuroectodermal tumor of infancy (Melanotic Progonoma) - an unsual soft tissue tumor. Zentralbl Alg Pathol 1983;128(5):307-16.

10. Dehner LP, Sibley RK, Sauk JJ. et al. Malignant neuroectodermal tumor of infancy. A clinical, pathological, ultrastructural and tissue culture study. Cancer 1976;43:1389-410.

11. Enzinger F, Weiss SW. Soft Tissue Tumors. 3rd edit. CV. Mosby, St. Louis 1995;879-81.

12. Schulz, DM. A Malignant Melanotic Neoplasm of the Uterus, Resembling the "Retinal Anlage" Tumors, Amer J Clin Path 1957;28:524.

13. Rodríguez-Galindo C, Marina N, Fletcher B, Parham D, Bodner S, Meyer W. Is Primitive Neuroectodermal Tumor of the Kidney a Distinct Entity? Cancer 1997;79(11): 2243-9.

14. Dilu NJ, Bone A, Sokolo MR. Melanotic Progonoma: A propos of a voluminous tumor in an infant. Rev Stomatol Chir Maxillofac 1998;99 (2):103-5.

15. Bardfield GH, Pleasants JE. Melanocitic neuroectodermic tumor of infancy. J Oral Surg 1976;34(9):839-841.

16. Navas Palacios JJ. Malignant melanotic neuroectodermic tumor: light and electron microscopic study. Cancer 1980;46:529-36.

17. Pettinato G, Manivel JC, D'Amore ES, Jaszac W, Gorlin RJ. Melanotic neuroectodermal tumor of 
infancy. A re-examination of histogenic problem based on inmunohistochemistry, flow citometry and ultrastructur study of 10 cases. Am J Surg Pathol 1991;15:233-245

18. Claros P, Claros A, Claros A Jr, Clavería A. Progonoma Melanótico del maxilar: Presentación de un caso. Acta Otorrinolaringol Esp. 1990;41 (2): 103-6.

\section{CORRESPONDENCIA}

Antonio Díaz Caballero

Facultad de Odontología. Universidad de Cartagena.

Campus de la Salud Zaragocilla.

Cartagena, Bolívar

Colombia

Correo electrónico: antoniodiazc@yahoo.com 